\title{
Anionic clay as the drug delivery vehicle: tumor targeting function of layered double hydroxide- methotrexate nanohybrid in C33A orthotopic cervical cancer model
}

\author{
This article was published in the following Dove Press journal: \\ International Journal of Nanomedicine \\ 20 January 2016 \\ Number of times this article has been viewed
}

\author{
Goeun Choil \\ Huiyan Piao' \\ Zeid A Alothman² \\ Ajayan Vinu ${ }^{3}$ \\ Chae-Ok Yun ${ }^{4}$ \\ Jin-Ho Choy' \\ 'Center for Intelligent Nano-Bio \\ Materials, Department of Chemistry \\ and Nano Science, Ewha Womans \\ University, Seoul, Korea; ${ }^{2}$ Advanced \\ Materials Research Chair, Chemistry \\ Department, College of Science, \\ King Saud University, Riyadh, Saudi \\ Arabia; ${ }^{3}$ Future Industries Institute, \\ University of South Australia, Mawson \\ Lakes, SA, Australia; ${ }^{4}$ Department \\ of Bioengineering, College of \\ Engineering, Hanyang University, \\ Seoul, Korea
}

\begin{abstract}
Methotrexate (MTX), an anticancer agent, was successfully intercalated into the anionic clay, layered double hydroxides to form a new nanohybrid drug. The coprecipitation and subsequent hydrothermal method were used to prepare chemically, structurally, and morphologically well-defined two-dimensional drug-clay nanohybrid. The resulting two-dimensional drug-clay nanohybrid showed excellent colloidal stability not only in deionized water but also in an electrolyte solution of Dulbecco's Modified Eagle's Medium with 10\% fetal bovine serum, in which the average particle size in colloid and the polydispersity index were determined to be around 100 and $0.250 \mathrm{~nm}$, respectively. The targeting property of the nanohybrid drug was confirmed by evaluating the tumor-to-blood and tumor-to-liver ratios of the MTX with anionic clay carrier, and these ratios were compared to those of free MTX in the C33A orthotopic cervical cancer model. The biodistribution studies indicated that the mice treated with the former showed 3.5-fold higher tumor-to-liver ratio and fivefold higher tumor-to-blood ratio of MTX than those treated with the latter at 30 minutes postinjection.
\end{abstract}

Keywords: anionic clay, biodistribution, cervical cancer, colloidal stability, layered double hydroxide, methotrexate

\section{Introduction}

Cancer is one of the most significant diseases worldwide due to its incidence, prevalence, and mortality, and for the past few decades, considerable efforts have been devoted to understanding the origin of cancer, developing early detection methods to improve the survival rate of patients, and designing novel theragnostic devices. In particular, nanomedicines have been suggested as a feasible alternative to treat cancer. Nanomedicine comprises of approximately six research fields such as drug delivery, drug and therapies, active implants, in vitro diagnostics, in vivo imaging, and biomaterials. ${ }^{1}$ However, the drug delivery is thought to be most intensively studied out of the six fields in terms of the total number of papers and patents published. ${ }^{2}$ To develop new nanoscale drug delivery system (DDS), nanosized drug delivery vehicles are required to have necessary and sufficient properties such as low toxicity, high-drug loading concentration, and excellent targeting function. Several DDS therapeutics based on nanomaterials such as liposomes, nanoparticles, and polymers have already been approved for clinical use in humans. However, there are still limitations in developing novel and efficient nanovehicles for chemotherapy applications. ${ }^{3,4}$ In our previous studies, two-dimensional minerals, such as anionic clay (hydrotalcite), could
Center for Intelligent Nano-Bio Materials, Department of Chemistry and Nano Science, Ewha Womans University, 52 Ewhayeodae-gil, Seodaemun-gu, Seoul 03760, Korea

Tel +82232774135

Fax +82 232774340

Email jhchoy@ewha.ac.kr 
have much competitiveness as drug delivery carriers over various organic and inorganic nanoparticles such as micelles, liposomes, polymersomes, dendrimers, polyphosphazenes, iron oxide, and porous silica including graphene oxides and carbon nanotubes, etc. ${ }^{3-5}$

In recent years, layered double hydroxide (LDH), which is nothing different from anionic clay with a hydrotalcite structure, has attracted an increasing amount of interest as a delivery nanovehicle owing to its high biocompatibility, controllable drug content, enhanced cellular permeation property, and ability to overcome drug resistance. ${ }^{6-12} \mathrm{LDH}$ has the chemical formula $\left[\mathrm{M}(\mathrm{II})_{1-\mathrm{x}} \mathrm{M}(\mathrm{III})_{\mathrm{x}}(\mathrm{OH})_{2}\right]^{\mathrm{x}+}\left(\mathrm{A}^{\mathrm{n}-}\right)_{\mathrm{x} / \mathrm{n}} \cdot \mathrm{mH}_{2} \mathrm{O}$ (where $\mathrm{M}(\mathrm{II})$ : divalent metal cations, $\mathrm{M}(\mathrm{III})$ : trivalent metal cations, $\mathrm{A}^{\mathrm{n}-}$ : anionic molecules, $0<\mathrm{x}<1$ ) and consists of positively charged layers and interlayer anions that are alternately stacked with each other along the crystallographic $c$-axis to form a 1:1 type heterostructure. ${ }^{13}$ The positive layer charge in LDH can be easily controlled by replacing the divalent cation, $\mathrm{M}(\mathrm{II})$, in the lattice partially with the trivalent one, $\mathrm{M}$ (III), in a way in which the negatively charged drug or biomolecules can be stabilized in the interlayer space of LDH to compensate for the positive layer charge. One thing to emphasize here is that the interlayer anion is exchangeable for LDH to intercalate various biologically active molecules, such as nucleotides, ${ }^{6-8}$ anticancer drugs, ${ }^{14,15}$ anti-inflammatory drugs, ${ }^{16,17}$ biopolymers, ${ }^{18,19}$ vitamins, ${ }^{20,21}$ and antibiotics ${ }^{22}$ into its two-dimensional lattice. Moreover, the anion content in LDH can be controlled because it depends directly on the magnitude of the anion exchange capacity, namely, that of the layer charge density. In recent years, nanosized LDHs with $\sim 100 \mathrm{~nm}$ were found to be biocompatible and could deliver drugs or gene molecules selectively to the tumor tissues and cells, owing to their intrinsic targeting function thanks to the enhanced permeability and retention effect and the clathrin-mediated endocytosis mechanism of LDHs. ${ }^{23-25}$ More recently, we successfully prepared the nanohybrid DDS, LDH-MTX, as a potential chemotherapeutic drug, where MTX stands for an anticancer drug, methotrexate (MTX; (2S)-2[(4-\{[(2,4-diamino-7,8-dihydropteridin-6-yl)methyl $]$ (methyl) amino \}phenyl)formamido]pentanedioic acid) (Figure S1), which has been prescribed for certain human cancers, including osteosarcoma, leukemia, and breast cancer and even for arthritis. Moreover, we found that the LDH-MTX nanohybrid could target tumor not only in vitro but also in vivo, as well demonstrated with an orthotopic breast cancer model. ${ }^{14}$

In this study, we attempted to apply the same strategy to the orthotopic model, which is considered to be clinically more relevant and consequently better predictive to infer drug efficacy and/or toxicity than the standard subcutaneous models. Because the tumor cells are implanted directly into the very organ, this model reflects the real-life situation (such as microenvironment) of cancer patient much better than the conventional xenograft tumor model. ${ }^{26}$ Among various female cancer models, such as ovarian-, breast-, and cervical cancers, ${ }^{27}$ we decided to prepare the orthotopic cervical cancer to finalize the nanohybrid DDS study based on the orthotopic breast cancer model. To the best of our knowledge, this is the first orthotopic cervical cancer model study to prove the drug delivery behavior of anionic clay after the injection.

The coprecipitation method and subsequent hydrothermal route were used to prepare the chemically, structurally, and morphologically well-defined nanohybrid drug LDH-MTX. The resulting LDH-MTX nanoparticles with colloidal property were characterized by powder X-ray diffraction (PXRD), dynamic light scattering, field emission scanning electron microscopy, and high-resolution transmission electron microscopy (HR-TEM) studies. The TEM and particle size distribution studies indicate that the colloidal LDH-MTX particles were homogeneously distributed with an average particle size of $\sim 100 \mathrm{~nm}$. According to our previous study, LDH nanoparticles in the size range of 100-200 nm could accumulate more in tumor tissues than in normal tissues, eventually resulting in effective tumor targeting surely owing to the enhanced permeability and retention effect. ${ }^{28-30}$ Moreover, the therapeutic efficacy of the present colloidal LDH-MTX nanohybrid in the orthotopic cervical cancer model was investigated and compared to that of free MTX. To confirm the targeting function of the nanohybrid drug, the tumor-to-blood and tumor-to-liver ratios of MTX with LDH carrier were evaluated and compared to those of free MTX in the present orthotopic model.

\section{Materials and methods Materials}

MTX $\left(\mathrm{C}_{20} \mathrm{H}_{22} \mathrm{~N}_{8} \mathrm{O}_{5}\right.$, purity $\left.>98.0 \%\right)$, magnesium chloride hexahydrate $\left(\mathrm{MgCl}_{2} \cdot 6 \mathrm{H}_{2} \mathrm{O}\right.$, purity $>98.0 \%$, pharmacopeia grade), and aluminum chloride hexahydrate $\left(\mathrm{AlCl}_{3} \cdot 6 \mathrm{H}_{2} \mathrm{O}\right.$, purity $>99.0 \%$, pharmacopeia grade) were purchased from Sigma-Aldrich Co. Ltd. (Korea). Decarbonated water was used for the entire synthesis procedure, and all reagents were used without further purification.

\section{Animals}

All the mice were kept in a laminar air flow cabinet under specific pathogen free conditions in Yonsei University. The animal facilities were permitted from an Association for Assessment and Accreditation of Laboratory Animal 
Care. All the animal experiments were conducted under the institutional guidelines established by the Animal Research Committee. The animal related protocols were approved by the Committee on the Ethics of Animal Experiments of Yonsei University.

\section{Preparation of the LDH-MTX nanohybrid} A colloidal LDH with the formula $\mathrm{Mg}_{0.667} \mathrm{Al}_{0.333}(\mathrm{OH})_{2}$ $\mathrm{Cl}_{0.333} \cdot 0.5 \mathrm{H}_{2} \mathrm{O}$ was prepared by the direct coprecipitation and subsequent hydrothermal treatment. An aqueous solution of $\mathrm{MgCl}_{2} \cdot 6 \mathrm{H}_{2} \mathrm{O}(0.066 \mathrm{M})$ and $\mathrm{AlCl}_{3} \cdot 6 \mathrm{H}_{2} \mathrm{O}(0.033 \mathrm{M})$ was titrated dropwise with $0.5 \mathrm{M} \mathrm{NaOH}$ solution under a nitrogen atmosphere, and the final solution $\mathrm{pH}$ was adjusted to be $9.5 \pm 0.2$. The resulting white precipitate was aged for 3 hours, collected by centrifugation, washed with deionized water, and then further treated under hydrothermal condition at $100^{\circ} \mathrm{C}$ for 6 hours to prepare colloidal LDH nanoparticles.

To prepare the LDH-MTX nanohybrid, MTX was first dispersed in deionized water and titrated with a $0.5 \mathrm{M} \mathrm{NaOH}$ solution to afford a $0.033 \mathrm{M}$ solution of MTX at $\mathrm{pH} 7.0 \pm 0.2$ to form a $\gamma$-carboxylate (Figure S1). ${ }^{31}$ To that solution, a mixed solution of $\mathrm{MgCl}_{2} \cdot 6 \mathrm{H}_{2} \mathrm{O}(0.066 \mathrm{M})$ and $\mathrm{AlCl}_{3} \cdot 6 \mathrm{H}_{2} \mathrm{O}$ $(0.033 \mathrm{M})$ was added dropwise, a $0.5 \mathrm{M} \mathrm{NaOH}$ solution was also added to maintain the $\mathrm{pH}$ at $9.5 \pm 0.2$, and the resulting slurry was further aged for 18 hours. The entire process was performed under a nitrogen atmosphere at room temperature. Finally, the resulting yellow precipitate was collected via centrifugation, washed with decarbonated water, dispersed in decarbonated water, and further hydrothermally treated at $100^{\circ} \mathrm{C}$ for 18 hours to prepare the desired colloidal LDHMTX nanohybrid.

\section{Characterization of the LDH-MTX nanohybrid}

For structural analysis, PXRD analyses were performed using a diffractometer (Rigaku D/MAX RINT 2200-Ultima+, Rigaku Corporation, Tokyo, Japan) with monochromatized and Ni-filtered $\mathrm{Cu} K_{\alpha}$ radiation $(\lambda=1.5418 \AA$ ), operating at $40 \mathrm{kV}$ and $30 \mathrm{~mA}$. The chemical compositions of the hybrids were determined by elemental (CHNS) analysis (EA1112, CE Instrument, Milan, Italy) and inductively coupled plasma-atomic emission spectrometry (Optima-4300 DV, PerkinElmer, Waltham, MA, USA). HR-TEM study was carried out using a JEOL JEM-2100F microscope (JEOL, Tokyo, Japan) operated at an accelerating voltage of $200 \mathrm{kV}$. The particle size distributions of LDH and LDH-MTX were measured using a zeta sizer (Nano ZS, Malvern, UK). The instrument parameters that were used to take measurements in the cell culture media are as follows: the refractive indices of the particles (2.09) and Dulbecco's Modified Eagle's Medium (DMEM) with 10\% fetal bovine serum (FBS) were 2.09 and 1.337 , respectively, and the temperature was $37^{\circ} \mathrm{C}$, and the dynamic viscosity of DMEM with $10 \%$ FBS was $0.738 \times 10^{-3}$ $\mathrm{Pa} \cdot \mathrm{s}^{32}$ The morphologies for LDH and LDH-MTX were studied by field emission scanning electron microscopy (JEOL JSM-6700F). The MTX content of the LDH-MTX nanohybrid was measured by high-performance liquid chromatography (HPLC) using an Agilent 1100 series HPLC system using a C18 column (Nucleosil, $250 \mathrm{~mm} \times 4.6 \mathrm{~mm}$, Alltech, Nicholasville, KY, USA) under the following condition: the mobile phase was $0.05 \mathrm{M} \mathrm{KH}_{2} \mathrm{PO}_{4}$ in $10 \%$ acetonitrile with the $\mathrm{pH}$ of 6.6 and the flow rate of $1 \mathrm{~mL} / \mathrm{min}$ and the column temperature of $40^{\circ} \mathrm{C}$. The MTX was detected by the UV absorption band at $304 \mathrm{~nm}$.

\section{Cell line and C33A orthotopic model}

Human cervical cancer cell (C33A) was obtained from Yonsei University Cancer Center, which was purchased from the American Type Culture Collection (ATCC, Manassas, VA, USA). The protocols for the cell line and C33A orthotopic model were approved by the Committee on the Ethics of Animal Experiments of Yonsei University. The cells were grown in DMEM medium (Welgene Ltd, Daegu, Korea) supplemented with $10 \% \mathrm{FBS}, 100$ units/mL penicillin, and $100 \mu \mathrm{g} /$ $\mathrm{mL}$ streptomycin at $37^{\circ} \mathrm{C}$ in a humidified atmosphere of $5 \%$ $\mathrm{CO}_{2}$. For the implantation of $\mathrm{C} 33 \mathrm{~A}$ cells, 7-week-old female athymic Balb/c nude mice (Orient Bio Inc., Gyeonggi-Do, Korea) were housed under controlled temperature, light, and humidity condition for 2 weeks before use. When the mice weighed in the range $20-22 \mathrm{~g}, \sim 2 \times 10^{6} \mathrm{C} 33 \mathrm{~A}$ cells in $30 \mu \mathrm{L}$ of phosphate-buffered saline (PBS) were inoculated in the mice uterus. Three weeks later, a laparotomy was performed to confirm that an orthotopic tumor had developed into a volume of $100-200 \mathrm{~mm}^{3}$.

\section{Antitumor activity in the orthotopic cervical cancer model}

The antitumor efficacy of LDH-MTX in mice with orthotopic C33A tumors was measured and compared to that of MTX only. When the tumor volume reached $\sim 100-200 \mathrm{~mm}^{3}$, the mice were randomly assigned into four groups ( $n=3$ per group) and injected intraperitoneally (ip) with PBS, LDH, MTX (50 mg $/ \mathrm{kg}$ ), or LDH-MTX (120 mg/kg, corresponding to $50 \mathrm{mg} / \mathrm{kg}$ MTX) once a week for 5 weeks, and their body weights were evaluated every $2-3$ days to evaluate the in vivo toxicity. Finally, the mice were sacrificed on the 4th day after the final treatment, and the tumor tissues were collected. Tumor size was measured using calipers, and 
the tumor volume was calculated based on the equation of volume $=$ length $\times$ width $^{2} \times 0.523$, where the constant 0.523 corresponds to the prolate ellipsoid formula. ${ }^{33,34}$

\section{Biodistribution studies}

The biodistribution of free MTX and LDH-MTX in the orthotopic cervical cancer model was compared by administering an ip injection of each drug PBS, MTX (60 mg/kg), or LDH-MTX (143 mg/kg, corresponding to $60 \mathrm{mg} / \mathrm{kg}$ MTX; $\mathrm{n}=3$ per group), and the mice were sacrificed at precisely 30 and 60 minutes after the ip injection. The samples were prepared for the biodistribution study by harvesting the organs and tumor tissues, homogenizing with four times the volume of $1 \mathrm{M} \mathrm{NaOH}$ solution, and centrifugation at 3,000 rpm for 10 minutes. Two volumes of acetonitrile were added to the ground samples and were vigorously mixed to obtain the protein precipitates. After centrifugation at 10,000 rpm for 3 minutes, four volumes of chloroform were added to the supernatant in order to remove the lipids. Finally, the supernatants were collected via centrifugation at 2,000 rpm for 10 minutes and were analyzed via HPLC (Agilent 1100 series Instrument, Agilent Technologies, Santa Clara, CA, USA). The MTX content in the cells was also quantified via HPLC.

\section{Inflammatory response test in mice}

To detect proinflammatory cytokine, tumor necrosis factor- $\alpha$ (TNF- $\alpha$ ) and interleukin-6 (IL-6), 5-week-old male Balb/c mice (18-20 g, Orient Bio Inc.) were grown under controlled temperature, humidity, and light condition for 1 week before use. In the case of free MTX, the dosage of $60 \mathrm{mg} / \mathrm{kg}$ was dissolved in PBS, but for LDH-MTX, $143 \mathrm{mg} / \mathrm{kg}$ dosage corresponding to the MTX dosage of $60 \mathrm{mg} / \mathrm{kg}$ was homogeneously dispersed in PBS. The mice (nine heads/group) were administered with an ip injection of each drug, and the corresponding plasmas were collected with respect to the given response time of 3,6 , and 12 hours to analyze TNF- $\alpha$ and IL- 6 levels by the enzyme-linked immune sorbent assay analysis. Enzyme-linked immunosorbent assays were conducted based on the manufacturer's instructions.

\section{Results and discussion}

\section{Characterization of the LDH-MTX nanohybrid}

The chemical compositions of pristine LDH and LDH-MTX determined by inductively coupled plasma were $\mathrm{Mg}_{0.667}$ $\mathrm{Al}_{0.333}(\mathrm{OH})_{2} \mathrm{Cl}_{0.333} \cdot 0.5 \mathrm{H}_{2} \mathrm{O}$ and $\mathrm{Mg}_{0.655} \mathrm{Al}_{0.344}(\mathrm{OH})_{2}\left[(\mathrm{MTX})_{0.107}\right.$ $\left.\mathrm{Cl}_{0.128}\right] \cdot 0.1 \mathrm{H}_{2} \mathrm{O}$, respectively (Table 1 ), where the MTX composition of 0.107 was derived from the content of MTX
Table I Physico-chemical properties of pristine LDH and LDH-MTX nanohybrid

\begin{tabular}{llll}
\hline & Chemical formula & $\begin{array}{l}\text { MTX } \\
\text { contents (\%) }\end{array}$ & $\begin{array}{l}\text { Particle } \\
\text { thickness (nm) }\end{array}$ \\
\hline Pristine & $\mathrm{Mg}_{0.667} \mathrm{Al}_{0.333}(\mathrm{OH})_{2} \mathrm{Cl}_{0.333} \cdot$ & - & 13.3 \\
$\mathrm{LDH}$ & $0.5 \mathrm{H}_{2} \mathrm{O}$ & & \\
$\mathrm{LDH}-$ & $\mathrm{Mg}_{0.655} \mathrm{Al}_{0.344}(\mathrm{OH})_{2}$ & $42.00 \pm 0.25$ & 19.4 \\
MTX & {$\left[(\mathrm{MTX})_{0.107} \mathrm{Cl} \mathrm{l}_{0.128} \cdot 0.1 \mathrm{H}_{2} \mathrm{O}\right.$} & & \\
\hline
\end{tabular}

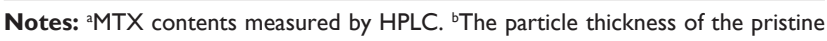
LDH and LDH-MTX nanohybrid samples was calculated from (00I) X-ray diffraction peaks by using Scherrer's formula, described by $t=0.9 \lambda / B \cos \theta$, where, $\lambda$ is $1.5418 \AA$ $\left(\mathrm{Cu} \mathrm{K}{ }_{\alpha}\right)$ and $B$ is FWHM at the diffraction angle of $\theta$. - represents 'zero' since the pristine LDH does not contain any MTX molecules.

Abbreviations: FWHM, full width at half maximum; LDH, layered double hydroxide; MTX, methotrexate; LDH-MTX, layered double hydroxide-methotrexate; HPLC, high-performance liquid chromatography.

$(42.00 \% \pm 0.25 \%)$ in LDH-MTX as quantitatively measured by HPLC. This result is in excellent agreement with that measured by the CHNS analysis. Figure 1 shows the PXRD patterns of MTX, LDH, and LDH-MTX. The pristine LDH exhibited a series of well-developed (001) reflections with a basal spacing of $7.69 \AA$. For LDH-MTX, the basal spacing was clearly increased to $21.0 \AA$ after the intercalation of MTX into the LDH lattice. Given $4.8 \AA$ as the single layer thickness, the gallery height of LDH-MTX became $16.2 \AA(21.0-4.8 \AA)$. The longitudinal and lateral molecular dimensions of MTX are 21.2 and $6.5 \AA$, respectively, so the MTX molecules in the interlayer of LDH are thought to be monolayer oriented with a tilted angle of $43^{\circ}$ relative to the basal plane..$^{14,35}$

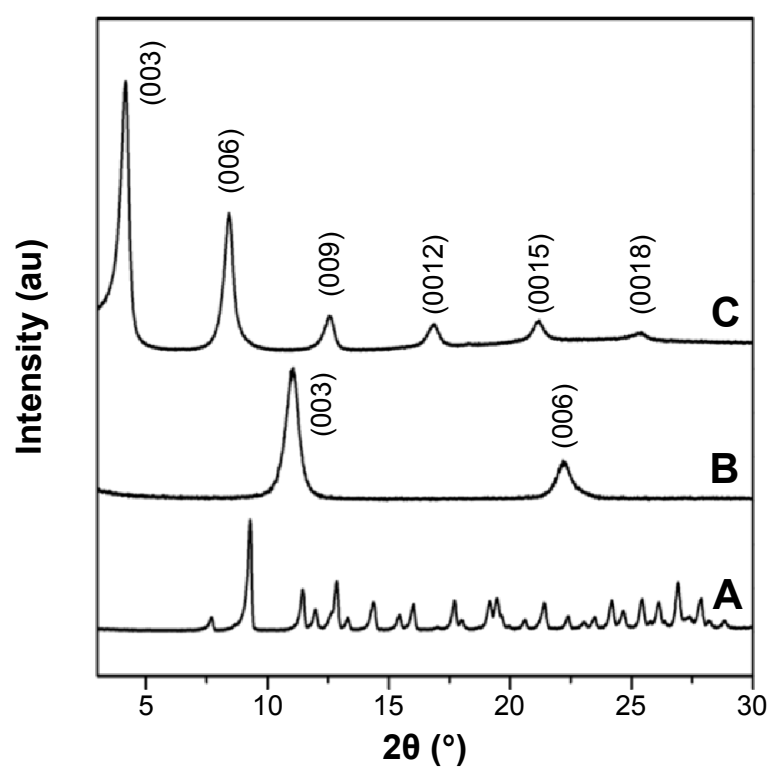

Figure I Powder X-ray diffraction (PXRD) patterns for (A) pure MTX, (B) pristine LDH, and (C) LDH-MTX nanohybrid.

Abbreviations: au, arbitrary unit; LDH, layered double hydroxide; MTX, methotrexate; LDH-MTX, layered double hydroxide-methotrexate. 
The cross-sectional HR-TEM images of the LDH-MTX nanohybrid clearly show a well-ordered layered structure (Figure 2). The interlayer distance, which corresponds to the periodic lattice fringe, was again confirmed to be 7.6 and $21.0 \AA$ for pristine LDH and LDH-MTX, respectively, and these values are in good agreement with the $d_{001}$ values of 7.69 and $21.0 \AA$ for the former and the latter, respectively. As shown in Figure 2A and C, the particle thickness for pristine LDH and LDH-MTX were $\sim 10$ and $20 \mathrm{~nm}$, respectively, those which are also well consistent with the calculated particle thicknesses of 13.3 and $19.4 \mathrm{~nm}$ derived from the (001) X-ray diffraction peaks by using Scherrer's formula, $t=0.9 \lambda / B \cos \theta$, where $\lambda$ is $1.5418 \AA\left(\mathrm{Cu} \mathrm{K}_{\alpha}\right)$ and $B$ is full width at half maximum at the diffraction angle of $\theta$ (Table 1). ${ }^{36}$

\section{Evaluation of colloidal property for LDH- MTX}

For in vitro and in vivo studies with LDH-MTX, preparing its colloidal suspension in buffer or cell culture media is required. In our previous studies, we reported on a colloid hybrid drug, "drug-ceramic 2-D nanoassemblies for DDS under physiological condition". ${ }^{37}$ However, the polydispersity index (PDI) value of LDH-MTX was still $>0.45$, where the PDI reflects the homogeneity of the colloid dispersion. The PDI values range from 0.0 for an entire homogeneous dispersion to 1.0 for a completely inhomogeneous one. ${ }^{38-40}$ Therefore, an investigation of the PDI for the colloidal stability of the LDH-MTX in serum was strongly required. In this study, different from the previous studies, we developed an ingenious way of preparing colloidal LDH nanoparticles; first, LDH precipitate was prepared by the coprecipitation, then completely washed with decarbonated water, and finally hydrothermally treated at $100^{\circ} \mathrm{C}$. The initial precipitate was not colloidal when dispersed in an aqueous solution due to the formation of aggregates. However, the particles then became colloidal when they were completely washed with decarbonated water to remove excess salts remaining in the solution and subsequently hydrothermally treated at $100^{\circ} \mathrm{C}$. In the case of anisotropic particles such as nanoclay, a lot of crystal edge sites are coordinatively unsaturated, and eventually reactive, resulting in the formation of aggregates. However,

\section{A}
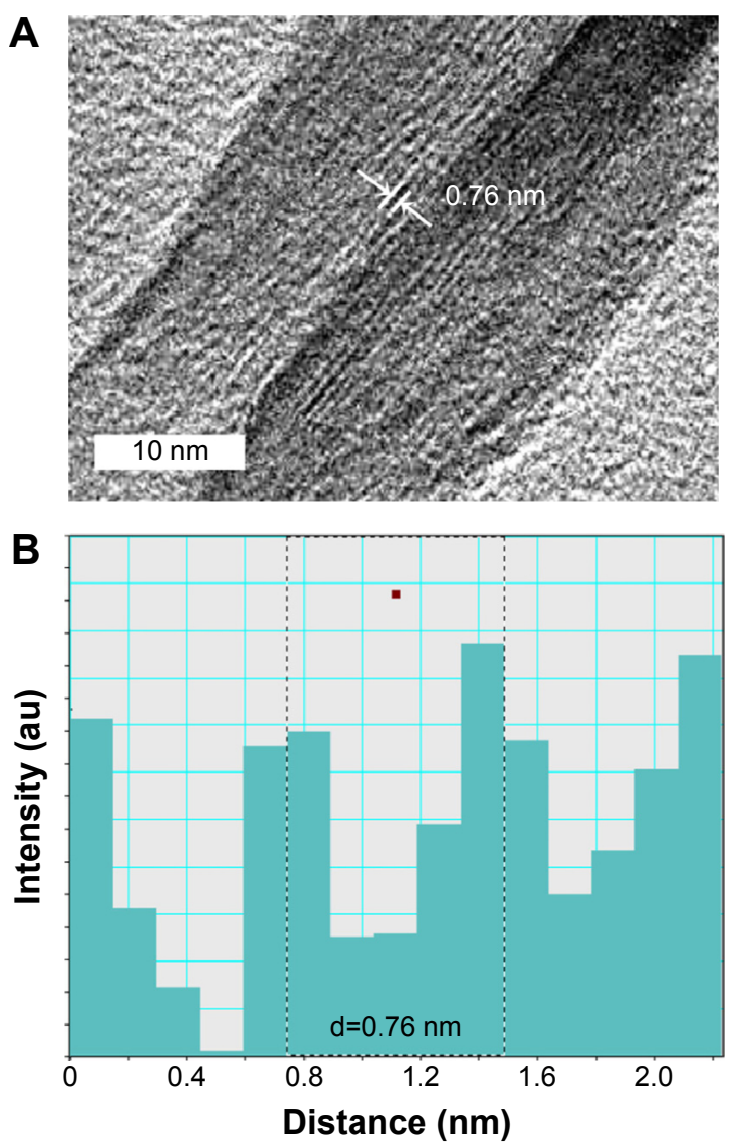

C

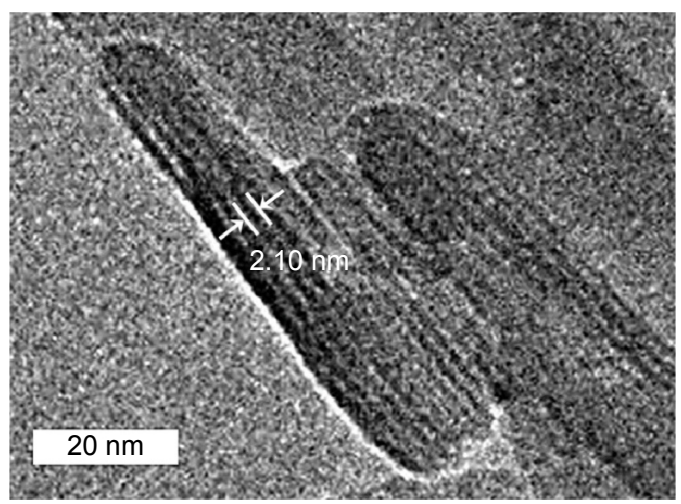

D

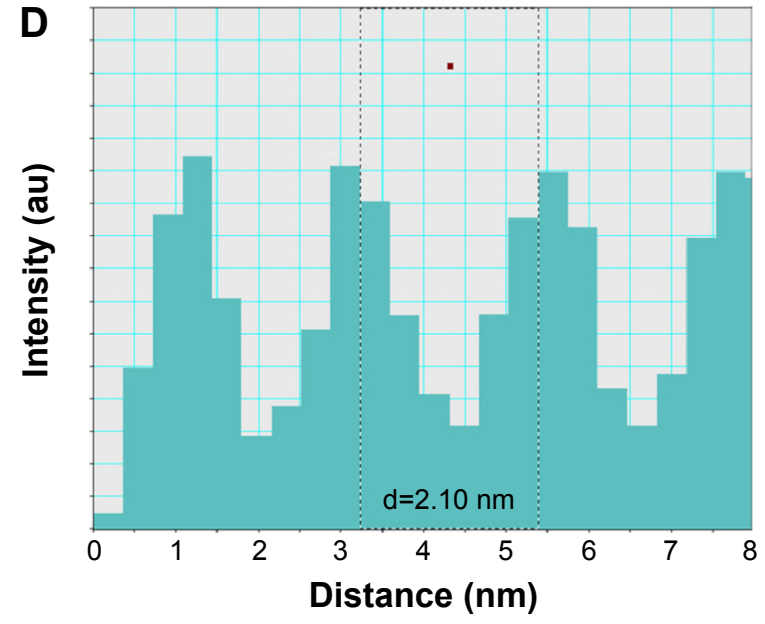

Figure 2 Cross-sectional high-resolution transmission electron microscopy images and intensity histogram of (A and $\mathbf{B})$ pristine LDH and (C and $\mathbf{D})$ LDH-MTX nanohybrid. Abbreviations: au, arbitrary unit; LDH, layered double hydroxide; LDH-MTX, layered double hydroxide-methotrexate. 
Table 2 Average particle size and PDI of pristine LDH and LDH-MTX nanohybrid

\begin{tabular}{llll}
\hline & Disperse media & $\begin{array}{l}\text { Particle } \\
\text { size }(\mathbf{n m})^{\mathbf{a}}\end{array}$ & PDI \\
\hline Pristine LDH & Distilled water & 71 & 0.107 \\
& DMEM with 10\% FBS & 86 & 0.254 \\
LDH-MTX & Distilled water & 95 & 0.110 \\
& DMEM with 10\% FBS & 104 & 0.250 \\
\hline
\end{tabular}

Note: aParticle size measured by the DLS method in suspension.

Abbreviations: DLS, dynamic light scattering; DMEM, Dulbecco's Modified Eagle's Medium; FBS, fetal bovine serum; LDH, layered double hydroxide; LDH-MTX, layered double hydroxide-methotrexate; PDI, polydispersity index.

under a high-temperature and high-pressure condition such as hydrothermal one, the edge sites could be coordinatively saturated and as a consequence deactivated, resulting in a stable colloid. As shown in Figures S2 and S3, the pristine LDH and LDH-MTX nanoparticles were fairly well dispersed in deionized water with an average particle size of 71 and $95 \mathrm{~nm}$, respectively, and their PDI values were determined to be 0.107 and 0.110 , respectively (Table 2 ). When each of LDH and LDH-MTX was added to an electrolyte solution of DMEM with 10\% FBS, a homogeneous dispersion was obtained with an average particle size of 86 and $104 \mathrm{~nm}$ with the PDI values of 0.254 and 0.250 , respectively (Table 2, Figure 3).

\section{In vivo antitumor activity and body weight changes in the orthotopic cervical cancer model}

To understand the therapeutic efficacy of LDH-MTX, a tumor growth inhibition test was performed in the $\mathrm{C} 33 \mathrm{~A}$ orthotopic model and compared to that of MTX, PBS, and LDH, as shown in Figure 4. In the case of MTX only, the tumor volume was suppressed to $2,940.0 \mathrm{~mm}^{3}$ from $5,350.3$ $\mathrm{mm}^{3}$, representing a reduction of $\sim 45.0 \%$ compared to the PBS-treated group. However, the antitumor effect of LDHMTX was determined to be much more significant than that of MTX only, with a mean tumor volume of $988.1 \mathrm{~mm}^{3}$ that represented a reduction of $81.5 \%$ and $66.4 \%$ in tumor volume relative to the PBS and MTX groups, respectively. Such a remarkable suppression of tumor growth for the LDH-MTX-treated group was also demonstrated by the digital image of the tumor tissues in mice and compared to
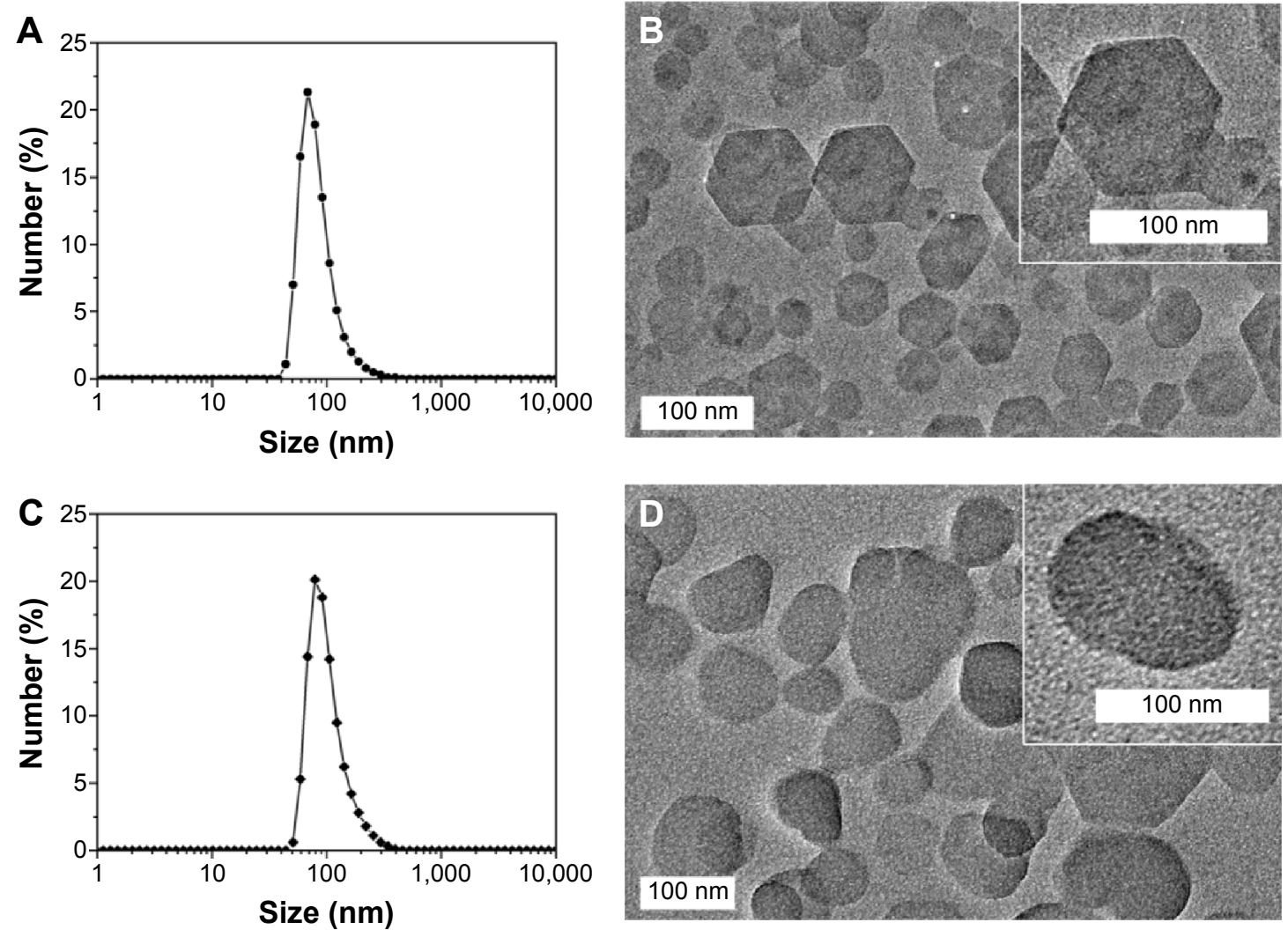

Figure 3 The particle size distribution and the high-resolution transmission electron microscopy images of (A and $\mathbf{B})$ pristine LDH and (C and $\mathbf{D})$ LDH-MTX in DMEM with 10\% FBS, respectively. The inset images (B and D) correspond to the enlarged images for pristine LDH and LDH-MTX, respectively.

Abbreviations: DMEM, Dulbecco's Modified Eagle's Medium; FBS, fetal bovine serum; LDH, layered double hydroxide; LDH-MTX, layered double hydroxide-methotrexate. 


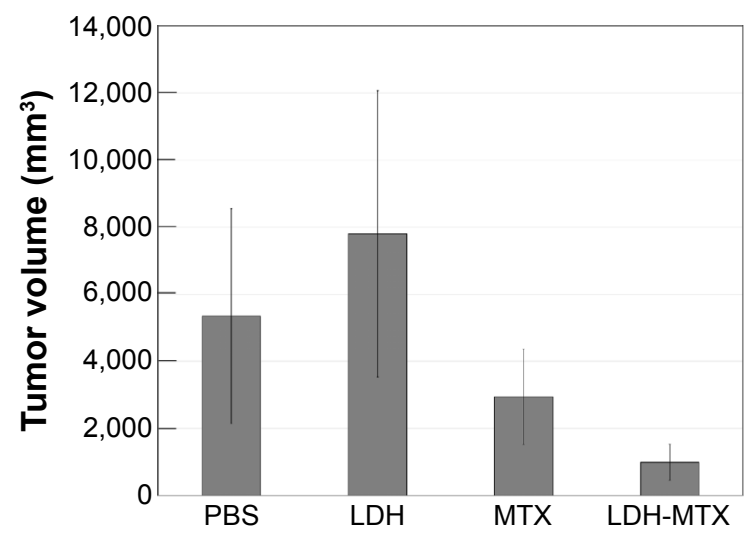

Figure 4 Antitumor activity of MTX and LDH-MTX in the C33A orthotopic cervical cancer model. PBS, LDH, MTX, and LDH-MTX were administered via intraperitoneal injection on days $0,7,14,21$, and 28 , and tumor volume was measured using calipers after 32 days.

Abbreviations: LDH, layered double hydroxide; MTX, methotrexate; LDH-MTX, layered double hydroxide-methotrexate; PBS, phosphate-buffered saline.

the PBS-, LDH-, and MTX-treated mice (Figure S4). To understand the in vivo toxicity, the body weight of the mice was regularly monitored after the injection of free MTX, LDH-MTX, and LDH nanoparticles, with that of the PBStreated mice as the negative control. As shown in Figure 5, no significant difference was observed among the groups.

\section{Tumor-to-liver ratio and tumor-to-blood ratio of LDH-MTX}

To evaluate the efficacy of MTX in the LDH-MTX nanohybrid system, the accumulation degree of MTX in various organs after 30 and 60 minutes of administration was quantified. As shown in Figure 6, the MTX concentration in the tumor for the LDH-MTX-treated (30 minutes: $110.0 \pm 27.5 \mu \mathrm{g} / \mathrm{g} ; 60$ minutes: $89.0 \pm 11.0 \mu \mathrm{g} / \mathrm{g}$ ) mice was

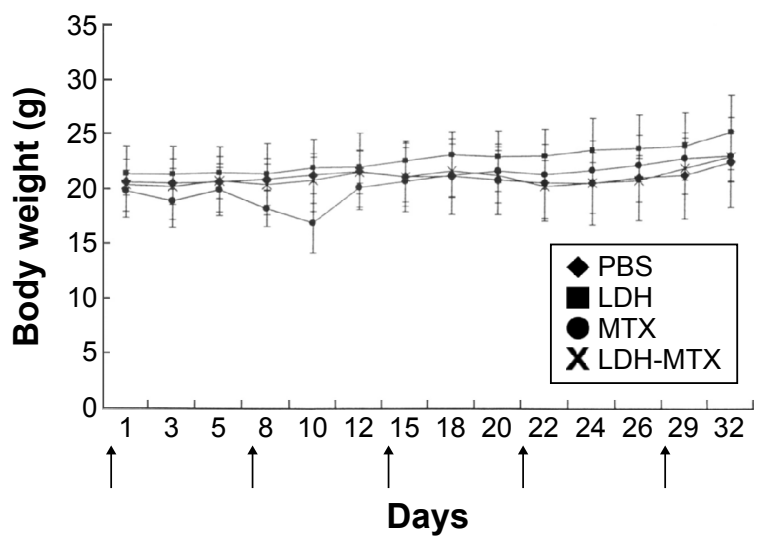

Figure 5 Body weight changes observed in the mice treated with PBS, LDH, MTX, and LDH-MTX.

Note: The arrows indicate the injection points.

Abbreviations: LDH, layered double hydroxide; MTX, methotrexate; LDH-MTX, layered double hydroxide-methotrexate; PBS, phosphate-buffered saline. considerably higher than that for free MTX (30 minutes: $30.0 \pm 3.0 \mu \mathrm{g} / \mathrm{g} ; 60$ minutes: $18.5 \pm 1.7 \mu \mathrm{g} / \mathrm{g}$ ), surely because of the fact that LDH-MTX nanoparticles were more effectively targeted to the tumor tissues than the intact MTX. This observation indicates that LDH has played an efficient role as a drug delivery carrier. As shown in Figure 6 and Table S1, the tumor-to-blood ratio of LDH-MTX at 30 minutes was surprisingly reached to 4.89 , which was approximately five times higher than that of free MTX (1.00), indicating the targeting function of LDH. One thing to note here is that no significant difference of MTX concentration in the brain, heart, lung, spleen, or muscle was observed between MTXtreated group and LDH-MTX-treated one. In other words, the therapeutic efficacy could be maximized without inadvertent side effects, including toxicity to other organs. Although the MTX concentration in the liver tissue of LDH-MTXtreated group was higher than that of the MTX-treated one at 60 minutes after administration, the tumor-to-liver ratios of the LDH-MTX-treated (30 minutes: 1.52; 60 minutes: $0.89)$ were also determined to be higher than those of the MTX only treated mice (30 minutes: $0.43 ; 60$ minutes: 0.44 ), corresponding to the 3.5 -fold and twofold increase, respectively. Because the tumor-to-liver ratio is an initial parameter to check therapeutic properties and safety, an enhancement of this ratio for the LDH-MTX-treated mice indicates that the present DDS strategy can be considered as a potential systematic chemotherapy, especially for cervical cancer. As previously confirmed, both LDH nanovehicle and LDHMTX nanohybrid did not induce any liver toxicity, in terms of alanine aminotransferase and aspartate aminotransferase levels and hematoxylin and eosin staining of liver tissues as well. ${ }^{14}$

\section{Inflammatory response of MTX and LDH-MTX nanohybrid}

Inflammation is the first response of the immune system, and inflammatory mediators, such as TNF- $\alpha$ and IL- 6 are secreted, when exposed to foreign substances, to enhance an immune response. Therefore, the effect of MTX and LDH-MTX hybrid on TNF- $\alpha$ and IL- 6 release in mice was evaluated. TNF- $\alpha$ and IL- 6 were detected after 3 hours in mice that had been administered MTX and LDH-MTX, but the amount was negligible within the range of error (Figure S5). Furthermore, no significant immune response was observed even after 6 and 12 hours of response time in the mice administered MTX and LDH-MTX, indicating nontoxicity. 

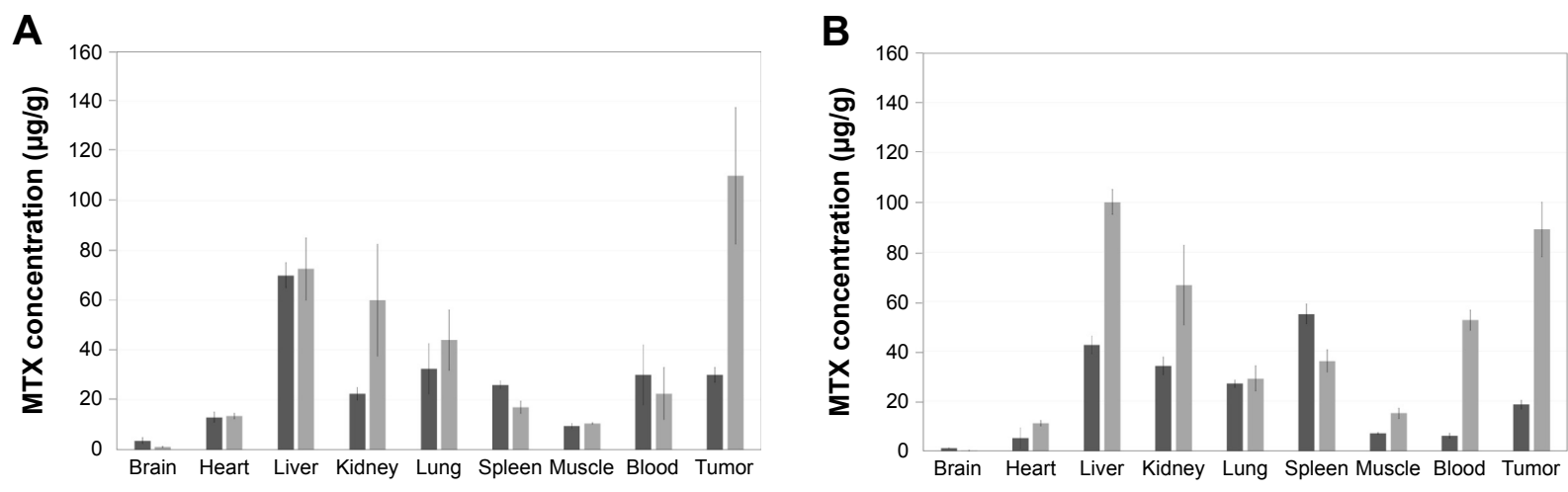

MTX LDH-MTX

Figure 6 Biodistribution studies of MTX in each tissue of C33A orthotopic tumor-bearing mice treated with MTX (dark gray) and LDH-MTX (gray) for (A) 30 minutes and (B) 60 minutes after administration.

Abbreviations: MTX, methotrexate; LDH-MTX, layered double hydroxide-methotrexate.

\section{Conclusion}

We have successfully developed colloidal LDH-MTX hybrid nanoparticles with an average particle size of $100 \mathrm{~nm}$ as an efficient DDS for chemotherapy. The biodistribution studies indicated that the LDH drug delivery vehicle exhibited a targeting effect that was quite evident because mice treated with LDH-MTX showed 3.5-fold higher tumor-to-liver ratio and fivefold higher tumor-to-blood ratio of MTX than those treated with free MTX at 30 minutes postinjection. The in vivo inflammatory response and body weight change profiles reveal that the incorporation of MTX into a nontoxic clay carrier (LDH) provides a novel nanohybrid DDS. Therefore, the present nanohybrid colloid with an average particle size of $100 \mathrm{~nm}$ can be proposed as a promising injectable chemotherapy due to its low in vitro and in vivo toxicities and high targeting effect giving rise to an enhanced therapeutic efficacy.

\section{Acknowledgments}

This work was supported by the National Research Foundation of Korea (NRF) Grant funded by the Korean Government (MSIP) (2005-0049412). G Choi is thankful to the Solvay Scholarship.

\section{Disclosure}

The authors report no conflicts of interest in this work.

\section{References}

1. Shi J, Votruba AR, Farokhzad OC, Langer R. Nanotechnology in drug delivery and tissue engineering: from discovery to applications. Nano Lett. 2010;10(9):3223-3230.

2. Wagner V, Dullaart A, Bock AK, Zweck A. The emerging nanomedicine landscape. Nat Biotech. 2006;24:1211-1217.
3. Yokoyama M, Okano T, Sakurai Y, Ekimoto H, Shibazaki C, Kataoka K. Toxicity and antitumor activity against solid tumors of micelle-forming polymeric anticancer drug and its extremely long circulation in blood. Cancer Res. 1991;51(12):3229-3236.

4. Parnham MJ, Wetzig H. Toxicity screening of liposomes. Chem Phys Lipids. 1993;64(1-3):263-274.

5. Choi SJ, Oh JM, Choy JH. Human-related application and nanotoxicology of inorganic particles: complementary aspects. J Mater Chem. 2008;18(6):615-620.

6. Choy JH, Kwak SY, Park JS, Jeong YJ, Portier J. Intercalative nanohybrids of nucleoside monophosphates and DNA in layered metal hydroxide. J Am Chem Soc. 1999;121(6):1399-1400.

7. Choy JH, Kwak SY, Jeong YJ, Park JS. Inorganic layered double hydroxides as nonviral vectors. Angew Chem Int Ed Engl. 2000;39(22): 4042-4045.

8. Desigaux L, Belkacem MB, Richard P, et al. Self-assembly and characterization of layered double hydroxide/DNA hybrids. Nano Lett. 2006;6(2):199-204.

9. Kwak SY, Kriven WM, Wallig MA, Choy JH. Inorganic delivery vector for intravenous injection. Biomaterials. 2004;25(28):5995-6001.

10. Choy JH, Jung JS, Oh JM, et al. Layered double hydroxide as an efficient drug reservoir for folate derivatives. Biomaterials. 2004; 25(15):3059-3064.

11. Choi SJ, Choi GE, Oh JM, Oh YJ, Park MC, Choy JH. Anticancer drug encapsulated in inorganic lattice can overcome drug resistance. J Mater Chem. 2010;20(42):9463-9469.

12. Khan AI, Lei L, Norquist AJ, O'Hare D. Intercalation and controlled release of pharmaceutically active compounds from a layered double hydroxide. Chem Commun. 2001;(22):2342-2343.

13. Cavani F, Trifirò F, Vaccari A. Hydrotalcite-type anionic clays: preparation, properties and applicatons. Catal Today. 1991;11(2):173-301.

14. Choi G, Kwon OJ, Oh Y, Yun CO, Choy JH. Inorganic nanovehicle targets tumor in an orthotopic breast cancer model. Sci Rep. 2014;4:4430; doi:10.1038/srep04430.

15. Choi SJ, Oh JM, Chung HE, Hong SH, Kim IH, Choy JH. In vivo anticancer activity of methotrexate-loaded layered double hydroxide nanoparticles. Curr Pharm Des. 2013;19(41):7196-7202.

16. Ribeiro LNM, Alcântara ACS, Darder M, Aranda P, Araújo-Moreira FM, Ruiz-Hitzky E. Pectin-coated chitosan-LDH bionanocomposite beads as potential systems for colon-targeted drug delivery. Int J Pharm. 2014;463(1):1-9.

17. Li B, He J, Evans DG, Duan X. Inorganic layered double hydroxides as a drug delivery system-intercalation and in vitro release of fenbufen. Appl Clay Sci. 2004;27(3-4):199-207. 
18. Darder M, López-Blanco M, Aranda P, Leroux F, Ruiz-Hitzky E. Bionanocomposites based on layered double hydroxides. Chem Mater. 2005;17(8):1969-1977.

19. Leroux F, Gachon J, Besse JP. Biopolymer immobilization during the crystalline growth of layered double hydroxide. J Solid State Chem. 2004;177(1):245-250.

20. Aisawa S, Higashiyama N, Takahashi S, et al. Intercalation behavior of L-ascorbic acid into layered double hydroxides. Appl Clay Sci. 2007;35(3-4):146-154.

21. Choy JH, Son YH. Intercalation of vitamer into LDH and their controlled release properties. Bull Korean Chem Soc. 2004;25(1):122-126.

22. Trikeriotis M, Ghanotakis DF. Intercalation of hydrophilic and hydrophobic antibiotics in layered double hydroxides. Int J Pharm. 2007;332(1-2):176-184.

23. Oh JM, Choi SJ, Kim ST, Choy JH. Cellular uptake mechanism of an inorganic nanovehicle and its drug conjugates: enhanced efficacy due to clathrin-mediated endocytosis. Bioconjugate Chem. 2006;17(6):1411-1417.

24. Oh JM, Choi SJ, Lee GE, Kim JE, Choy JH. Inorganic metal hydroxide nanoparticles for targeted cellular uptake through clathrin-mediated endocytosis. Chem Asian J. 2009;4(1):67-73.

25. Choi SJ, Choy JH. Layered double hydroxide nanoparticles as targetspecific delivery carriers: uptake mechanism and toxicity. Nanomedicine. 2011;6(5):803-814.

26. Killion JJ, Radinsky R, Fidler IJ. Orthotopic models are necessary to predict therapy of transplantable tumors in mice. Cancer Metastasis Rev. 1999;17(3):279-284.

27. Siegel R, Naishadham D, Jemal A. Cancer statistics, 2013. CA Cancer J Clin. 2013;63(1):11-30.

28. Maeda H. SMANCS and polymer-conjugated macromolecular drugs: advantages in cancer chemotherapy. Adv Drug Deliv Rev. 2001; 46(1-3):169-185.

29. Maeda H, Sawa T, Konno T. Mechanism of tumor-targeted delivery of macromolecular drugs, including the EPR effect in solid tumor and clinical overview of the prototype polymeric drug SMANCS. J Control Release. 2001;74(1-3):47-61.
30. Torchilin VP. Targeted pharmaceutical nanocarriers for cancer therapy and imaging. AAPS J. 2007;9(2):E128-E147.

31. Poe M. Acidic dissociation constants of folic acid, dihydrofolic acid, and methotrexate. J Biol Chem. 1977;252(11):3724-3728.

32. Khanbeigi RA, Kumar A, Sadouki F, et al. The delivered dose: applying particokinetics to in vitro investigations of nanoparticle internalization by macrophages. J Control Release. 2012;162(2):259-266.

33. Nagell JR, Gallion HH, Pavlik EJ, DePriest PD. Ovarian cancer screening. Cancer. 1995;76(10):2086-2091.

34. Riccabona M, Nelson TR, Pretorius DH. Three-dimensional ultrasound: accuracy of distance and volume measurements. Ultrasound Obstet Gynecol. 1996;7:429-434.

35. Kim JY, Choi SJ, Oh JM, Park T, Choy JH. Anticancer drug-inorganic nanohybrid and its cellular interaction. J Nanosci Nanotechnol. 2007;7(11):3700-3705.

36. Cullity BD. Elements of X-ray Diffraction. 2nd ed. Menlo Park, CA: Addison-Wesley; 1978.

37. Choi G, Kim SY, Oh JM, Choy JH. Drug-ceramic 2-dimensional nanoassemblies for drug delivery system in physiological condition. $J$ Am Ceram Soc. 2012;95(9):2758-2765.

38. Kallumadil M, Tada M, Nakagawa T, Abe M, Southern P, Pankhurst QA. Suitability of commercial colloids for magnetic hyperthermia. J Magn Magn Mater. 2009;321(10):1509-1513.

39. Siekmann B, Bunjes H, Koch MH, Westesen K. Preparation and structural investigations of colloidal dispersions prepared from cubic monoglyceride-water phases. Int J Pharm. 2002;244(1-2):33-43.

40. Cherng JY, Talsma H, Verrijk R, Crommelin DJ, Hennink WE. The effect of formulation parameters on the size of poly-((2-dimethylamino) ethyl methacrylate)-plasmid complexes. Eur J Pharm Biopharm. 1999;47(3):215-224. 


\section{Supplementary materials}<smiles>CN(Cc1cnc2nc(N)nc(N)c2n1)c1ccc(C(=O)N[C@@H](CCC(=O)O)C(=O)O)cc1</smiles>

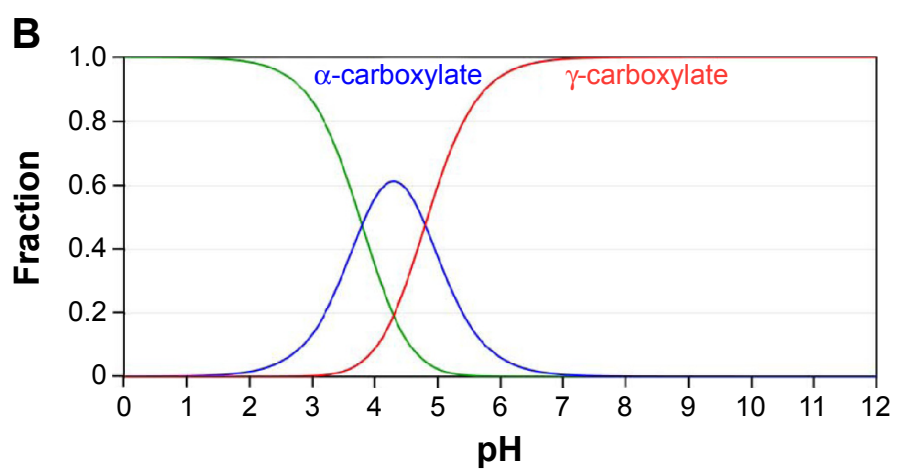

Figure SI (A) Molecular structure of methotrexate (MTX; (2S)-2-[(4-\{[(2,4-diamino-7,8-dihydropteridin-6-yl)methyl](methyl)amino\}phenyl)formamido]pentanedioic acid), and (B) plots of MTX molar fractions vs $\mathrm{pH}\left(\mathrm{pK}_{\mathrm{a} 1}=3.8, \mathrm{pK}_{\mathrm{a} 2}=4.8\right) .1$

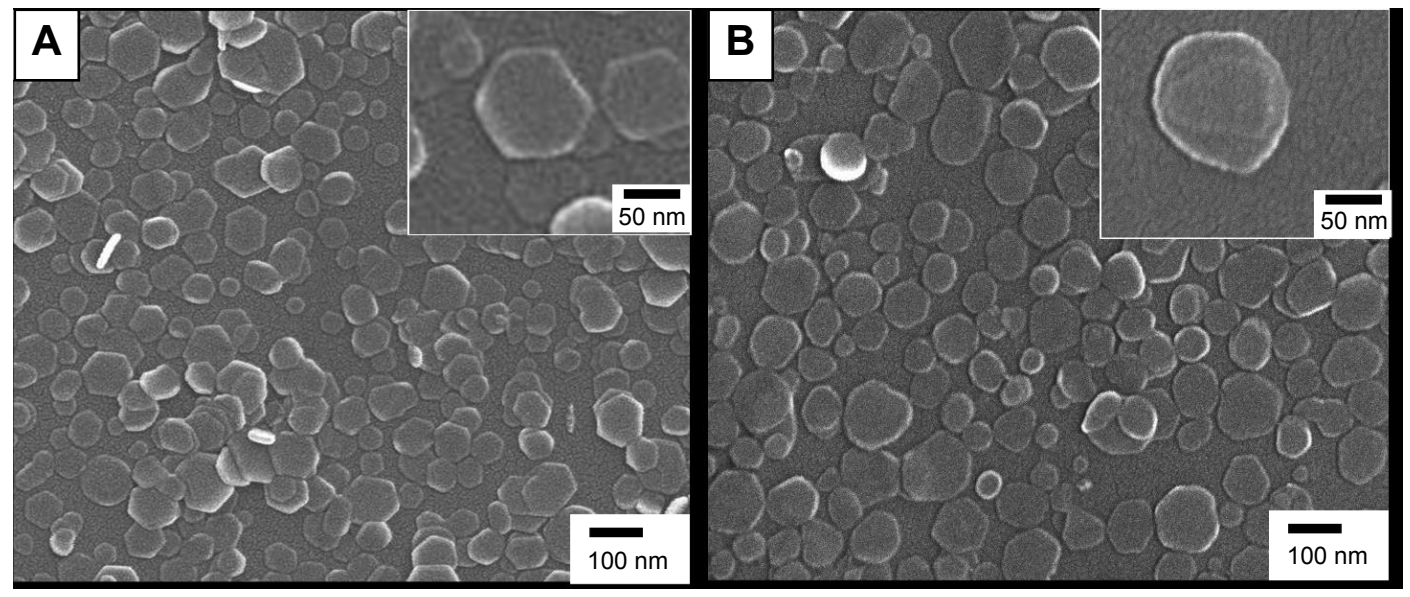

Figure S2 Scanning electron microscopic images of (A) pristine LDH, and (B) LDH-MTX nanohybrid in distilled water, respectively. The inset images (A and B) correspond to the enlarged images for pristine LDH and LDH-MTX, respectively.

Abbreviations: LDH, layered double hydroxide; LDH-MTX, layered double hydroxide-methotrexate. 
A

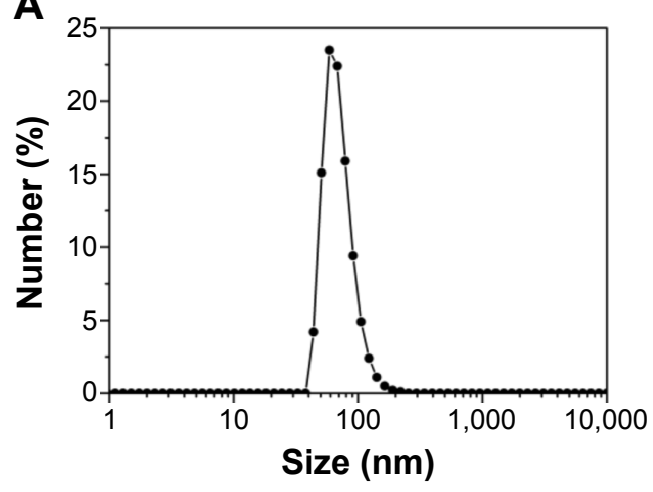

C

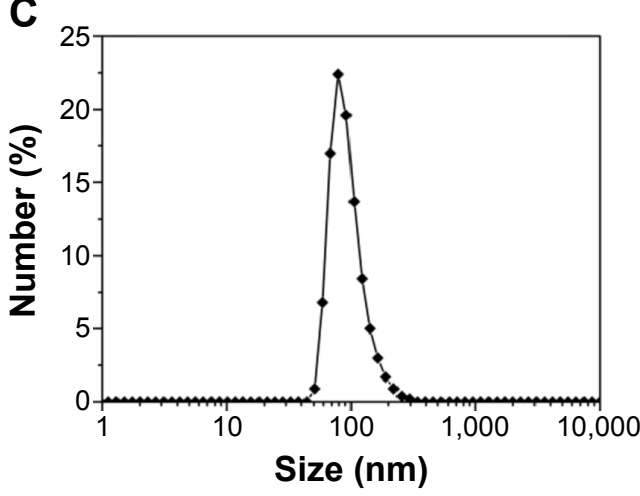

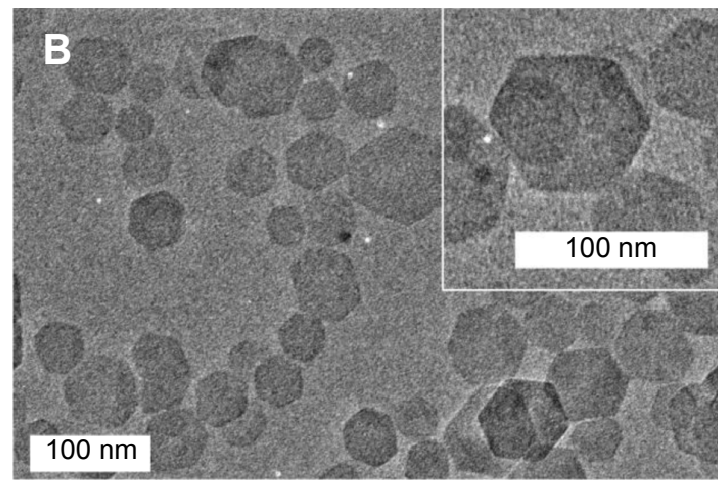

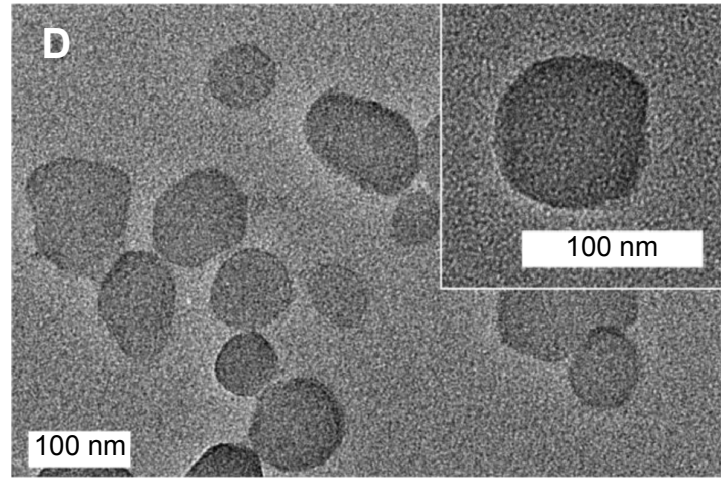

Figure S3 The particle size distribution and the high-resolution transmission electron microscopy images of (A and $\mathbf{B})$ pristine LDH, and (C and $\mathbf{D})$ LDH-MTX in distilled water, respectively. The inset images ( $\mathbf{B}$ and $\mathbf{D}$ ) correspond to the enlarged images for pristine LDH and LDH-MTX, respectively.

Abbreviations: LDH, layered double hydroxide; LDH-MTX, layered double hydroxide-methotrexate.

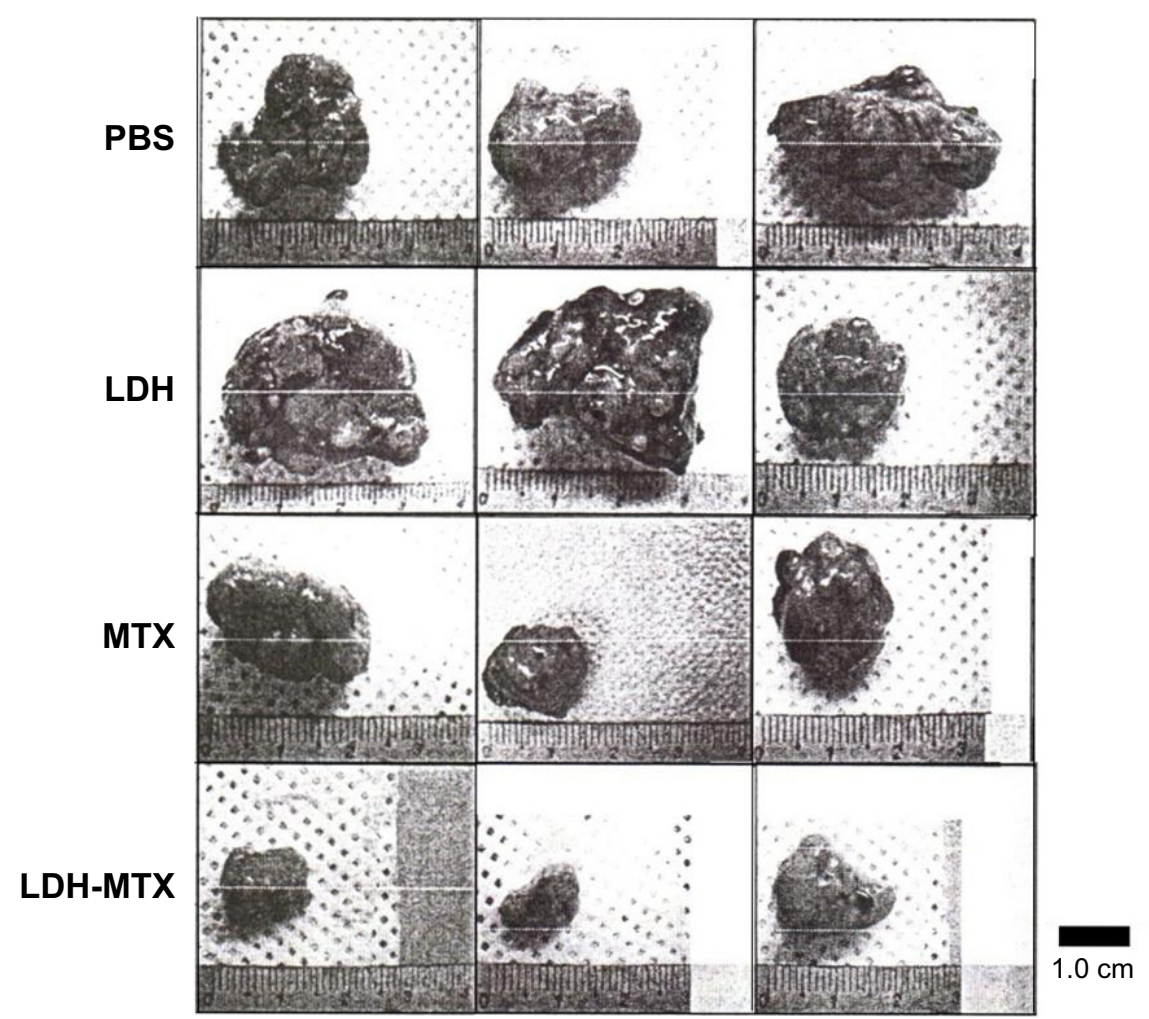

Figure S4 Digital images of tumor tissue in mice treated with PBS, LDH, MTX, and LDH-MTX. PBS, LDH, MTX, and LDH-MTX were administered via intraperitoneal injection on days $0,7,14,21$, and 28 , and tumor tissues were collected on 32 days.

Abbreviations: LDH, layered double hydroxide; MTX, methotrexate; LDH-MTX, layered double hydroxide-methotrexate; PBS, phosphate-buffered saline. 

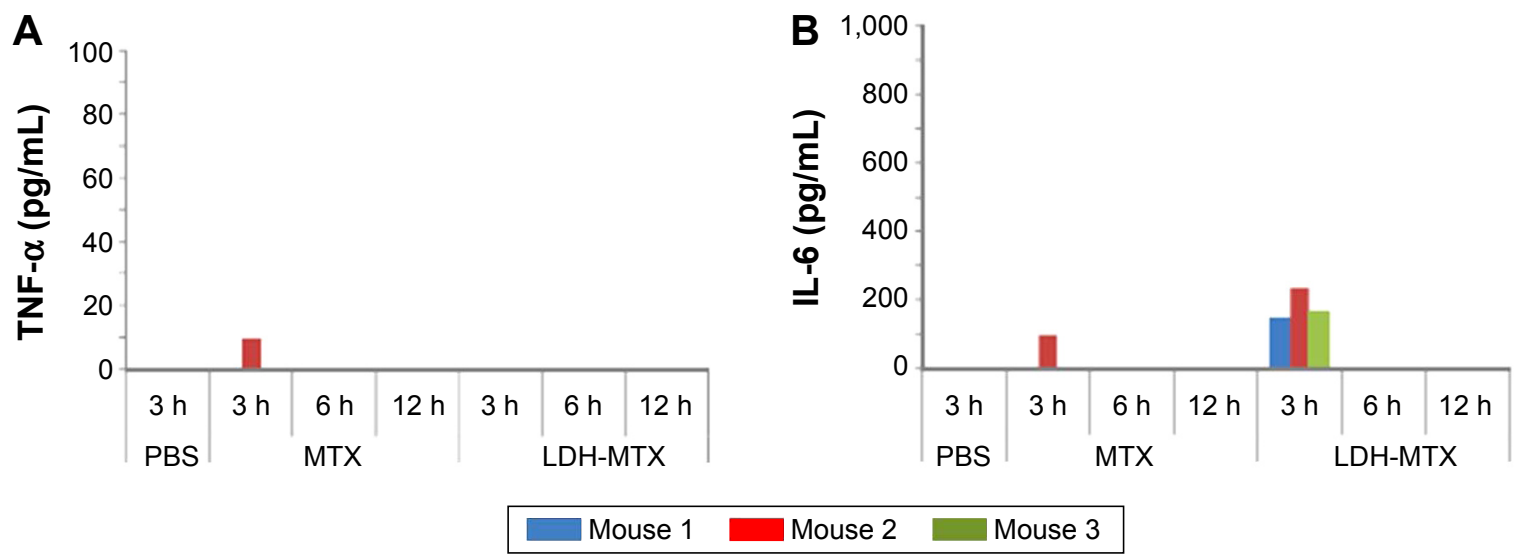

Figure S5 Immune response of MTX and LDH-MTX nanohybrids on the production of proinflammatory cytokine, (A) TNF- $\alpha$ and (B) IL-6.

Abbreviations: h, hours; IL-6, interleukin-6; MTX, methotrexate; LDH-MTX, layered double hydroxide-methotrexate; PBS, phosphate-buffered saline; TNF- $\alpha$, tumor necrosis factor- $\alpha$.

Table SI Tumor-to-liver and tumor-to-blood ratio of MTX in MTX-treated group and LDH-MTX-treated one

\begin{tabular}{lllll}
\hline Group & Tumor-to-liver & & Tumor-to-blood \\
\cline { 2 - 4 } & $\mathbf{3 0}$ minutes & $\mathbf{6 0}$ minutes & $\mathbf{3 0}$ minutes & $\mathbf{6 0}$ minutes \\
\hline MTX & 0.43 & 0.44 & 1.00 & 3.08 \\
LDH-MTX & 1.52 & 0.89 & 4.89 & 1.70 \\
\hline
\end{tabular}

Abbreviations: MTX, methotrexate; LDH-MTX, layered double hydroxide-methotrexate.

\section{Reference}

1. Poe M. Acidic dissociation constants of folic acid, dihydrofolic acid, and methotrexate. J Biol Chem. 1977;252(11):3724-3728.

\section{Publish your work in this journal}

The International Journal of Nanomedicine is an international, peerreviewed journal focusing on the application of nanotechnology in diagnostics, therapeutics, and drug delivery systems throughout the biomedical field. This journal is indexed on PubMed Central, MedLine, CAS, SciSearch $\AA$, Current Contents $\AA /$ Clinical Medicine,
Journal Citation Reports/Science Edition, EMBase, Scopus and the Elsevier Bibliographic databases. The manuscript management system is completely online and includes a very quick and fair peer-review system, which is all easy to use. Visit http://www.dovepress.com/ testimonials.php to read real quotes from published authors. 